\title{
Working Title: Clinician Engagement in Feedback Informed Treatment (FIT) and Patient Outcomes
}

\author{
Authors: \\ Jeb Brown, Ph.D, Center for Clinical Informatics \\ Christophe Cazauvieilh, Ph.D, IRPPSY and University of Bordeaux, France \\ Abstract: Aim, Methods, Results, Discussion
}

Aim: This paper presents analyses of outcome data for 317 therapists treating 14,161 patients over a three-year period to determine if therapists' effect sizes increased over time. Each therapist treated at least 5 patients in each of their first two years of using outcome measures. Multiple outcome questionnaires were employed. All measures also included a brief alliance scale administered concurrently.

Method: A severity adjusted effect size was calculated for each patient using intake scores and diagnostic group as predictors. The mean severity adjusted effect size for each therapist was calculated for their first and second years of using the outcome tools. This was done using a hierarchical linear model to control for sample size in each year, with a minimum sample of 5 cases in each year. Therapist engagement in receiving feedback was measured by counting the number of times the therapist logged into the online platform to view their results in each of the two years.

Results: Therapists who logged in the view their data at least 24 times in the second year $(n=123$; $37 \%$ ) averaged .92 effect size compared to .82 effect size for those seen by therapists who reviewed their results less frequently $(n=214 ; 63 \%)$. Login frequency during the first year was not predictive of effect size during the second year.

Discussion: The data provides evidence that effect sizes can trend upwards with measurement and feedback. Therapists' engagement in receiving feedback appears to increase the likelihood of effect size gain. 


\section{Introduction}

The purpose of this brief report is to present findings from the ongoing exploration of the relationship between clinicians engagement in receiving feedback from routine measurement and patient outcomes within the same time period. Clinician engagement was measured by the frequency with which clinicians logged into the ACORN Toolkit to view their data.

The report is intended to present findings in a simple format with easy to interpret graphs to assist is visualizing the distribution of results. More detailed reporting on results are available upon request.

The report is primarily intended to participants within the ACORN Collaboration and assumes a familiarity with the web bases application ACORN Devicision Support Toolkit and the metrics used to report clinical outcomes.

\section{Background}

Over the past decade the ACORN collaboration has developed and validated a methodology for benchmarking behavavioral health treatment outcomes as measuremented by pre-post change on a variety of client/patient completed outcome questionnaires assessing symptoms of depression, anxiety, social conflict/isolation, functioning in daily tasks and other symptoms treated through outpatient behavioral health services. The benchmarking methodology makes use of a standardized change score (Cohen's d) commonly reffered to ass effect size. This permits combing results from multiple questionnaires providing that the questionnaires share a common underlying construct as determined using factor analysis. (Cohen, J., 1988; Minami et al, 2007; Miami et al, 2008a; Minami et al, 2008b; Minami et al, 2009; Minami et al, 2012)

Prior analyses conducted in collaboraition with the ACORN Scientific Advisory Committee has reveal an association between how frequently a clinicians view their data and the distribution of the patient outcomes as evaluated using the Severity Adjusted Effect Size (SAES). SAES controls for differences in severity of distress at intake, diagnositic group, and session number of the first assessment on the treatment episode. (Brown, Simon, Cameron \& Minami, 2015)

Tracking clinician effectiveness as well as the frequency of Toolkit usage over a period of several years has revealed the outcomes tend to vary with the concurrent use of the Toolkit. For example, a clinician with excellent results in one year who then tends to log in much less often the the following year is more likely to see results go down compared to a clinician who continues to $\log$ in with adequeat frequency.

The present set of analyses is intended to demonstrate with phenomena through use of easy to understand results supported by graphs.

\section{Selection criteria}

A sample of therapists was identified based on two criteria: 
1) a sample of at least 100 clinical range cases with multiple assessments prior to January 30,2018

2) at least 15 similar cases in the 12 months following.

The estimated effect size for each of the therapist for cases prior to January 19, 2018 was calculated using the ACORN methodology for evaluating therapists' outcomes. The mean Severity Adjusted Effect Size (SAES) is calculated for each therapist, using Hierarchical Linear Modeling to adjust for sample size. For a more complete explanation of these methods and results, see Are You Any Good as a Therapist? (Brown, Simon \& Minami, 2015)

A total of 317 clinicians met these criteria. The mean SAES (calculated at the therapists' level) for this sample of therapists was .83 for the past 12 months, and .82 for all years prior to that. By way of comparison, the mean SAES for all cases in the ACORN database during the past three year is $.82(\mathrm{n}=159,989)$. This indicates that the outcomes for the group of clinicians included in the sample is comparable to the larger sample of ACORN participating clinicians.

The sample was divided into three groups of effectiveness:

1. low effectiveness (results in lower $25 \%$; effect size $<.72 ; \mathrm{N}=75$ )

2. average effectiveness (results in middle $50 \% ; \mathrm{N}=160$ )

3. high effectiveness (results in upper $25 \%$; effect size $>.91 ; \mathrm{N}=82$ )

In order to investigate the predictive validity of these therapists' rankings, a sample of their clinical range cases who were treated during 2018 were selected for further analyses $(n=14,638)$. The mean SAES for this sample is .835 .

As expected, the therapists estimated mean SAES prior to 2018 was significantly correlated with their clients' effect sizes in 2018 (Pearson $\mathrm{r}=.15, \mathrm{p}<.0001$ ). The following graph displays the mean effect sizes for patients treated by the groupings of therapists during the most recent 12 months. The differences in mean SAES and large $(p<.0001)$ and clinically meaningful. 


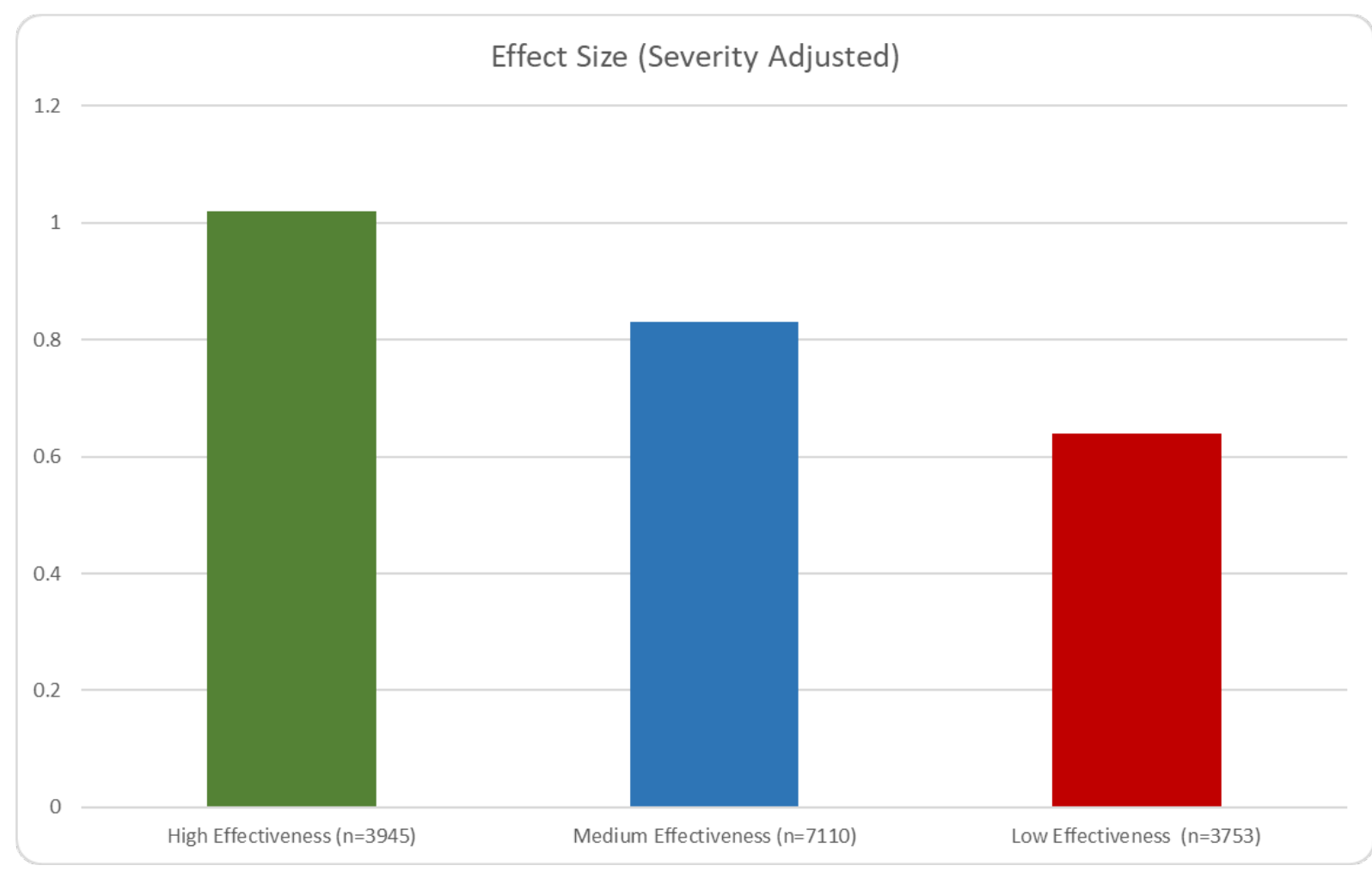

High Effectiveness ( $\mathrm{n}=3945$ )

Medium Effectiveness ( $\mathrm{n}=7110)$

Low Effectiveness $(\mathrm{n}=3753)$

\section{Effect Size}

(Severity

Adjusted)

Standard \% of

1.02

Deviation sample

0.83

$1.08 \quad 27.0 \%$

0.64

$1.1 \quad 47.4 \%$

$1.1 \quad 25.6 \%$

The following graph displays the distribution of individual client effect sizes for the three different groups of therapists. A client at the $50^{\text {th }}$ percentile for SAES treated by a clinician in the High Effectiveness group has a better result than about $58 \%$ of these treated by the Medium Effectiveness group and $64 \%$ treated by the Low Effectiveness group. 


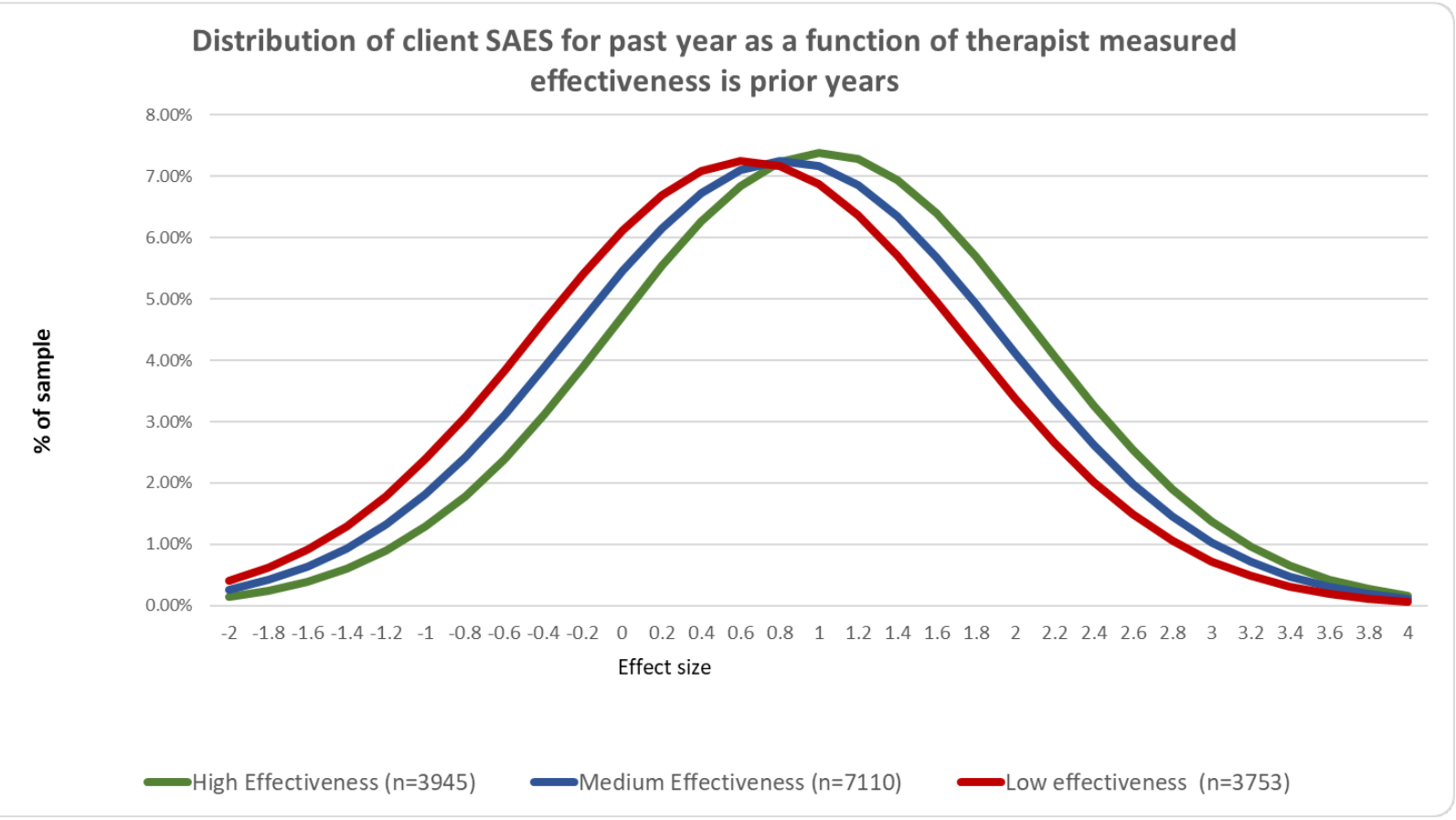

\section{Therapist engagement in feedback informed care}

Therapist engagement in receiving feedback on results was assessed by the frequency with which therapists logged into the Toolkit to view results. Prior analyses have revealed that this is not a linear relationship...more logins do not necessarily result in better outcomes. Rather, there appears to be a threshold effect, login in enough results in better outcomes but logging in much more does not result in even better results. On the other hand, logging in below the threshold frequency is associated with worse results, and it doesn't matter if the clinician never logged on or only logged in infrequently.

Prior analyses revealed that the threshold for benefit appears to be approximately 24 logins per year, or an average of twice a month. Based in this criterium, 148 (47\%) of the clinicians were classified as High Engagement while 169 (53\%) were classified a Low engagement. During the past year, those clinicians classified as High Engagement were significantly more effective during the past year, averaging .19 greater SAES. This both highly statistically significant $(\mathrm{p}<.0001)$ and clinically meaningful. 


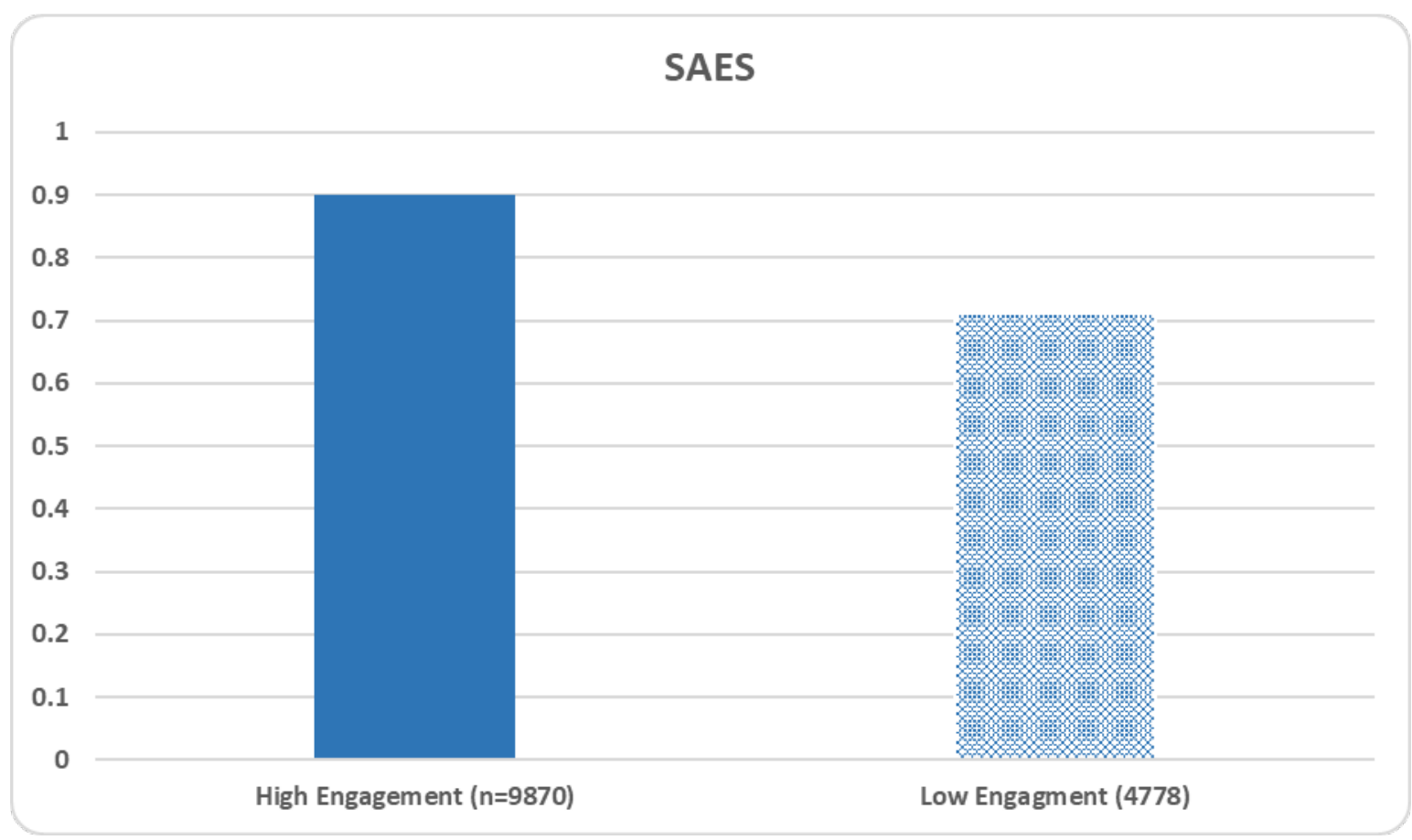

$\begin{array}{lcc} & & \text { Standard } \\ & \text { SAES } & \text { Deviation } \\ \text { High Engagement }(\mathrm{n}=9870) & 0.9 & 1.13 \\ \text { Low Engagement }(\mathrm{n}=4778) & 0.71 & 1.04\end{array}$

Note that High Engagement clinicians accounted for over two thirds of the sample in the past year, indicating that there is a significant association between the number of cases for which they have data and their likelihood of being highly engaged.

The following graph displays the distribution of results for individual cases. A client at the $50^{\text {th }}$ percentile in the High Engagement clinician group had a better outcome than about $57 \%$ of clients in the Low Engagement group. 


\section{Distribution of SAES as a function of therapists engagement in FIT}

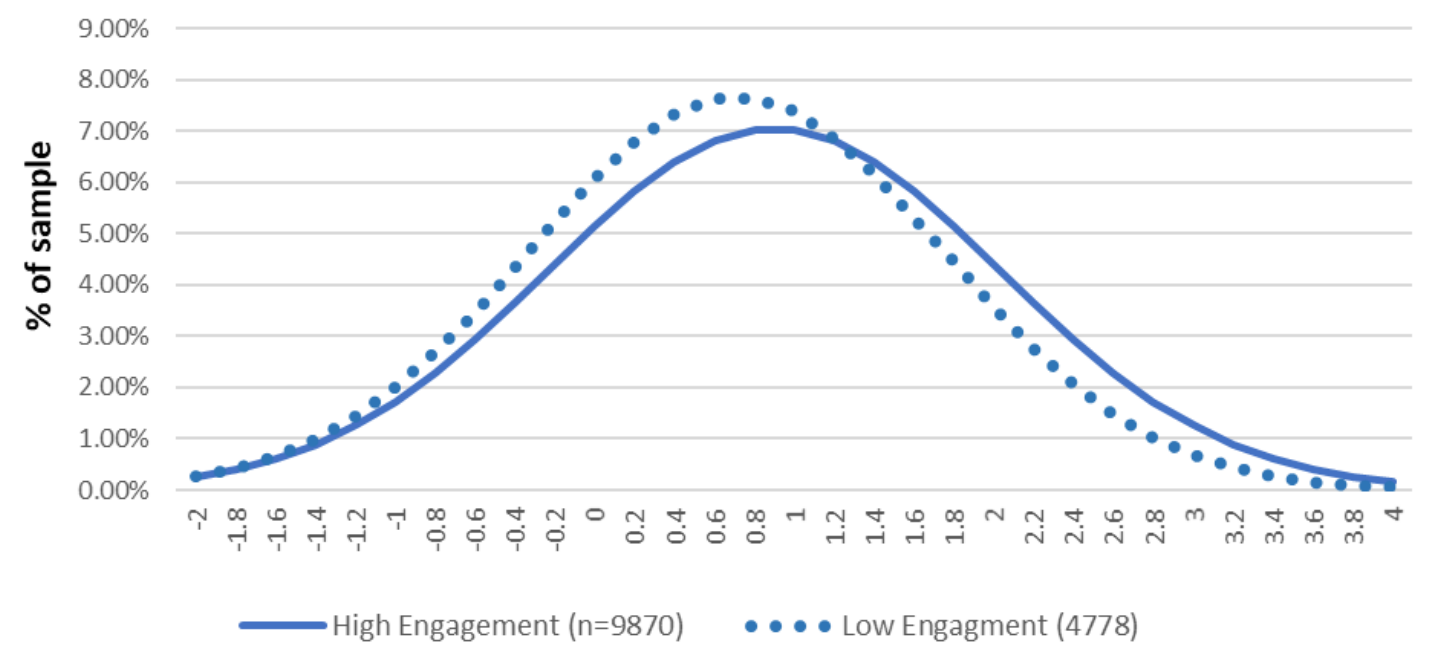

The breakdown on engagement varied greatly by clinician effectiveness group. For the High Effectiveness group, $84 \%$ of the cases were seen by high engaged clinicians, whereas in the Low Effectiveness group, only $46 \%$ were treated by highly engaged clinicians.

The differences in SAES between High Engagement and Low Engagement clinicians within each of the effectiveness groups were significant $(p<.05)$. The differences between High Effectiveness -Low Engagement and Medium Effectiveness-High Engagement, as well as between Medium Effectiveness-Low Engagement and Low Effectiveness -High Engagement did not reach this level of significance. This dramatically illustrates in impact of Engagement in FIT during the current year, regardless of level of Effectiveness is prior years. 


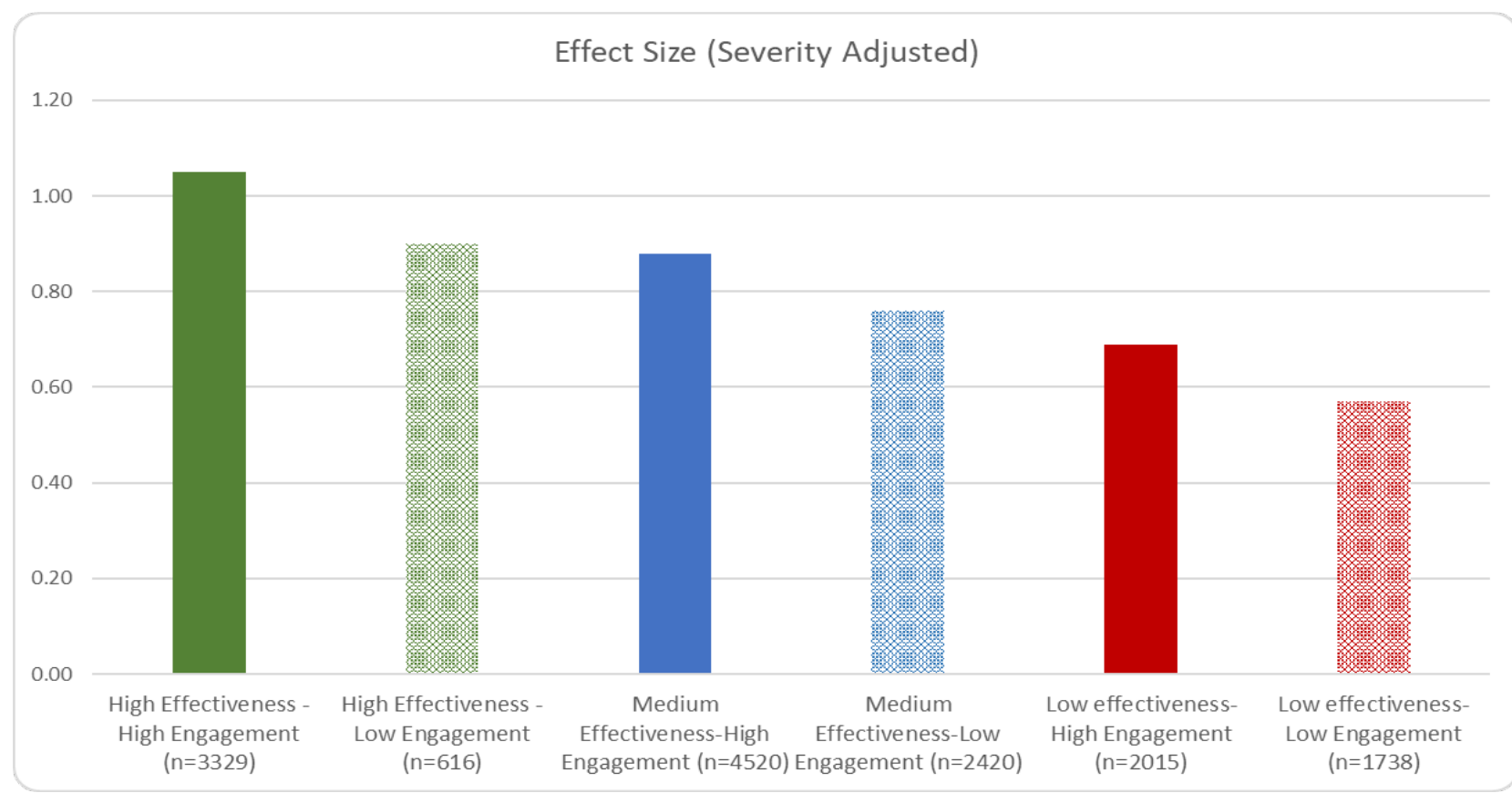

High Effectiveness -High Engagement ( $\mathrm{n}=3329)$

SAES Deviation

High Effectiveness -Low Engagement $(n=616)$

1.05

1.09

Medium Effectiveness-High Engagement $(n=4520)$

0.9

0.98

Medium Effectiveness-Low Engagement $(n=2420)$

0.88

1.13

Low effectiveness-High Engagement $(n=2015)$

0.76

1.04

Low effectiveness-Low Engagement $(n=1738)$

0.69

1.15

0.57

1.04

The following graph displays the distribution of clients' outcomes based on engagement during the past 12 months. Note that high engagement is associated with larger standard deviations. Better outcomes are not being achieved by reducing variability in outcomes, but rather by shifting the distribution in a positive direction while increasing the variation. 


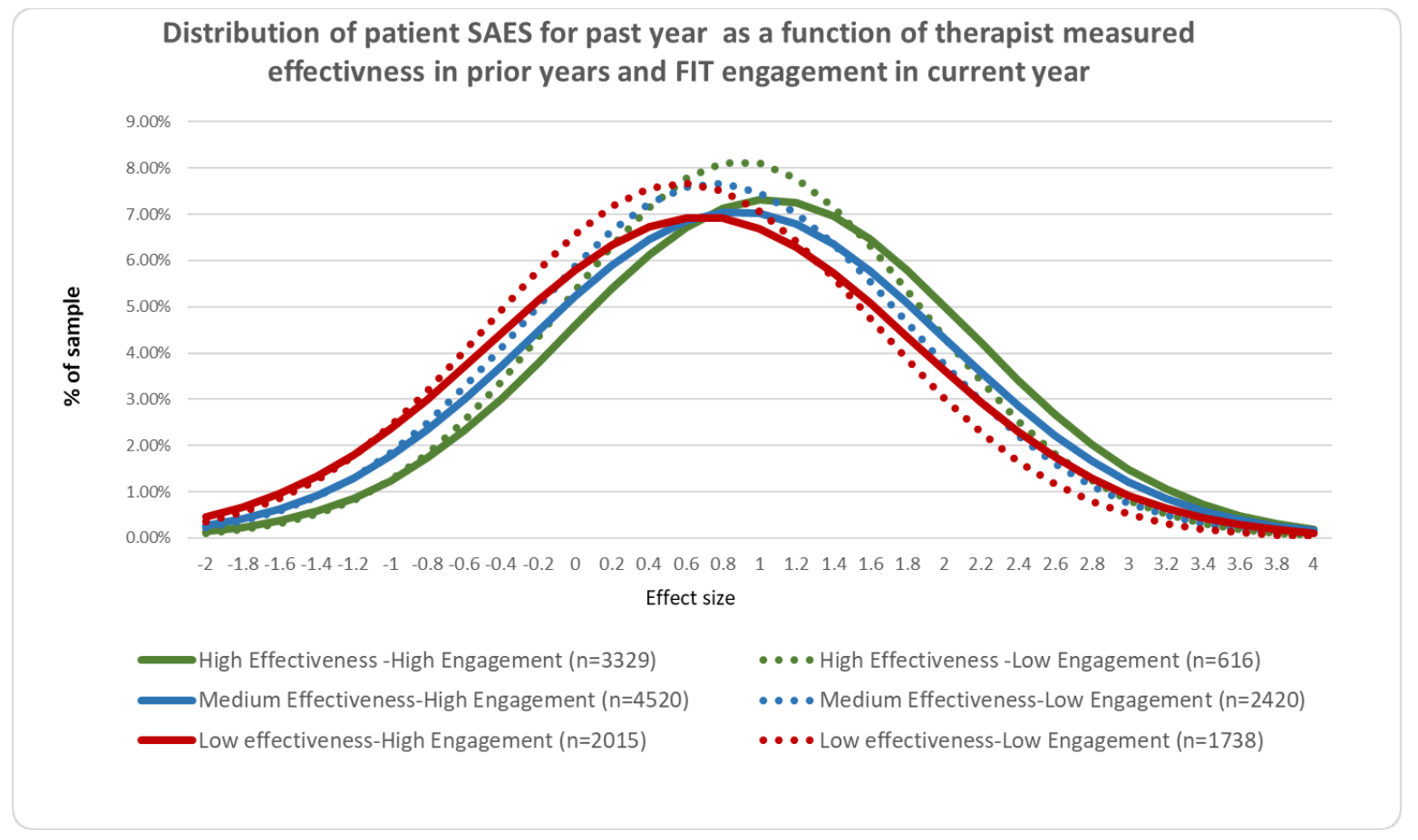

\section{Discussion}

This dataset provides compelling evidence that clinician engagement in receiving feedback on outcomes is associated with significantly larger effect sizes of clients. However, the mechanism for this effect is unclear.

Clinician engagement was measured simply by counting a behavior...how often did the clinician $\log$ into the website to view their data. Clinician engagement was voluntary, though incentives varies from location to location. Some clinics encouraged and monitored clinician engagement to a larger extent than others, and typical had higher levels of engagement.

One take way from these analyses is that it doesn't matter how effective a clinician believes they are, continued engagement in FIT is associated with better outcomes.

The unanswered question is why the results for highly engaged clinician are better. What are they doing differently, beyond simply logging into look at results?

To better understand what clinicians do once logged in, the ACORN Toolkit application was enhanced this past year to track page views and individual patient graphing views in more detail. However, it is to early to draw any conclusions from this data.

One feature of the ACORN Toolkit is the clinician are provided continuous feedback on their overall effectiveness and well as information on individual cases. It perhaps should not be surprising that real time performance feedback results in better performance. This continues to beg the question, what are they doing with the information. 


\section{Next Steps}

A logical next step would be to simply ask clinicians how they are using the information. Towards this end, the suggest creating a clinician completed questionnaire regarding their Toolkit usage, their thoughts on the information provided, and how they use the information.

Over the coming months we propose to reach out to ACORN participants and other potential academic collaborators to formulate a clinician completed questionnaire which can be used explore the clinicians' understanding of the value to of the information and how it is used in the clinical work. This is necessary step in further understanding how to best to assist clinicians to improve their personal effectiveness.

\section{References}

Brown, G. S. (J.), Simon, A., Cameron, J., \& Minami, T. (2015). A collaborative outcome resource network (ACORN): Tools for increasing the value of psychotherapy. Psychotherapy, 52(4), 412-421. https://doi.org/10.1037/pst0000033

Brown, G. S. J., Simon, A., \& Minami, T. (2015, April). Are you any good...as a therapist? [Web article]. Retrieved from http://www.societyforpsychotherapy.org/are-you-anygood-as-a-therapist

Cohen, J. (1988). Statistical power analysis for the behavioral sciences (2nd ed.). Hillsdale, N.J: Lawrence Erlbaum Associates.

Minami, T., Brown, G. S., McCulloch, J., \& Bolstrom, B. J. (2012). Benchmarking therapists: Furthering the benchmarking method in its application to clinical practice. Quality and Quantity, 46, 1699-1708. doi:10.1007/s11135-011-9548-4

Minami, T., Davies, D. R., Tierney, S. C., Bettmann, J. E., McAward, S. M., Averill, L. A., Huebner, L. A., Weitzman, L. M., Benbrook, A. R., Serlin, R. C., \& Wampold, B. E. (2009). Preliminary evidence on the effectiveness of psychological treatments delivered at a university counseling center. Journal of Counseling Psychology, 56, 309-320. doi:10.1037/a0015398

Minami, T., Serlin, R. C., Wampold, B. E., Kircher, J. C., \& Brown, G. S. (2008a). Using clinical trials to benchmark effects produced in clinical practice. Quality and Quantity, 42, 513525. doi:10.1007/s11135-006-9057-z

Minami, T., Wampold, B. E., Serlin, R. C., Kircher, J. C., \& Brown, G. S. (2007). Benchmarks for psychotherapy efficacy in adult major depression. Journal of Consulting and Clinical Psychology, 75, 232-243. doi:10.1037/0022-006X.75.2.232

Minami, T., Wampold, B. E., Serlin, R. C., Hamilton, E. G., Brown, G. S., \& Kircher, J. C. (2008b). Benchmarking the effectiveness of psychotherapy treatment for adult depression in a managed care environment: A preliminary study. Journal of Consulting and Clinical Psychology, 76, 116- 124. doi:10.1037/0022-006X.76.1.116 
